\title{
Effects of the polysaccharide nucleic acid fraction of bacillus Calmette-Guérin on the production of interleukin-2 and interleukin-10 in the peripheral blood lymphocytes of patients with chronic idiopathic urticaria
}

\author{
NA LI ${ }^{1}$, NA CAO $^{2}$, YAN-DONG NIU ${ }^{2}$, XIU-HUI BAI ${ }^{2}$, JIE LU $^{2}$, YU SUN $^{1}$, \\ MIN YU $^{1}$, LI-XIN SUN ${ }^{1}$ and XIN-SUO DUAN ${ }^{2}$ \\ ${ }^{1}$ Central Laboratory and ${ }^{2}$ Department of Dermatology, The Affiliated Hospital of Chengde Medical College, \\ Chengde, Hebei 067000, P.R. China
}

Received April 18,2013; Accepted July 03, 2013

DOI: 10.3892/br.2013.130

\begin{abstract}
Urticaria is one of the most frequent dermatoses and its prevalence in the general population is estimated to be $\sim 20 \%$, whereas a substantial percentage of the cases may be classified as chronic idiopathic urticaria (CIU). The inflammatory response presenting with spontaneous wheals exhibits pro-inflammatory characteristics, involving a prominent role for lymphocytes with a mixed Th1/Th2 response in which interleukin (IL)-2 and IL-10 are prominently secreted by Th1 and Th2 cells, respectively. In CIU patients, it was demonstrated that IL-10 production was elevated and IL-2 reduced compared to controls. Therefore, inhibition of IL-10 and promotion of IL-2 production by the lymphocytes, indicating Th2 inhibition and Th1 promotion, may facilitate the treatment of CIU. Whether the polysaccharide nucleic acid fraction of bacillus Calmette-Guérin (BCG-PSN), which possesses multiple immunomodulatory properties, has that potential, remains to be elucidated. In this study, BCG-PSN was used on lymphocytes isolated from CIU patients, with healthy donors used as controls. Immunocytochemistry and ELISA were used to detect IL-2 and IL-10 production. It was demonstrated that the IL-2 production by the lymphocytes in the CIU group was significantly lower compared to that in the healthy control group and it increased sequentially with the increase
\end{abstract}

Correspondence to: Dr Xin-Suo Duan, Department of Dermatology, The Affiliated Hospital of Chengde Medical College, 36 Nanyingzi Da Jie, Chengde, Hebei 067000, P.R. China

E-mail: duanxinsuo2002@163.com

Dr Li-Xin Sun, Central Laboratory, The Affiliated Hospital of Chengde Medical College, 36 Nanyingzi Da Jie, Chengde, Hebei 067000, P.R. China

E-mail: cyfyzys@163.com

Key words: polysaccharide nucleic acid fraction of bacillus Calmette-Guérin, chronic urticaria, lymphocyte, interleukin-2, interleukin-10 of the concentration of BCG-PSN used. By contrast, the IL-10 production by the lymphocytes in the CIU group was significantly higher compared to that in the healthy control group and decreased sequentially with the increase of the concentration of BCG-PSN used. Thus, it may be concluded that the BCG-PSN has the potential to promote IL-2 and inhibit IL-10 production in the lymphocytes of CIU patients, facilitating the treatment of CIU.

\section{Introduction}

Urticaria is a skin disorder presenting with localized edema due to vasodilatation and increased permeability of the small blood vessels in the mucosae and skin. The chronic form is defined as symptoms lasting for a minimum of 6 weeks. Chronic urticaria (CU) is characterized by recurrent, transitory, itchy wheals, which occur daily or almost daily (1). Several conditions are associated with allergy and autoimmune disorders, as well as urticaria, in which the clinical symptoms may be attributed to the release of histamine and other vasoactive mediators induced by the binding of a specific allergen to the IgE antibodies conjugated to the mast cell surface receptors $(2,3)$. However, in a substantial percentage of cases, the allergic trigger cannot be determined and the urticaria is classified as idiopathic (CIU), whereas $\sim 35-40 \%$ of cases appear to be of autoimmune origin $(4,5)$. The inflammatory response presenting with spontaneous wheals exhibits pro-inflammatory characteristics, involving a prominent role for lymphocytes with a mixed Th1/Th2 response (6). It was demonstrated that IL-10 production was elevated and IL-2 reduced in CIU patients compared to controls (7).

Certain natural products, such as the polysaccharide nucleic acid fraction of bacillus Calmette-Guérin (BCG-PSN), may possess immunoregulatory properties (8-10). BCG-PSN may increase $\mathrm{CD}^{+} \mathrm{T}$ cells and induce Th1-type immunity (11) and, by contrast, restrain Th2-type immune response, switching the balance of Th1/Th2 towards the Th1 side (12-15). A recent study by Luo et al (16) demonstrated that BCG priming and 

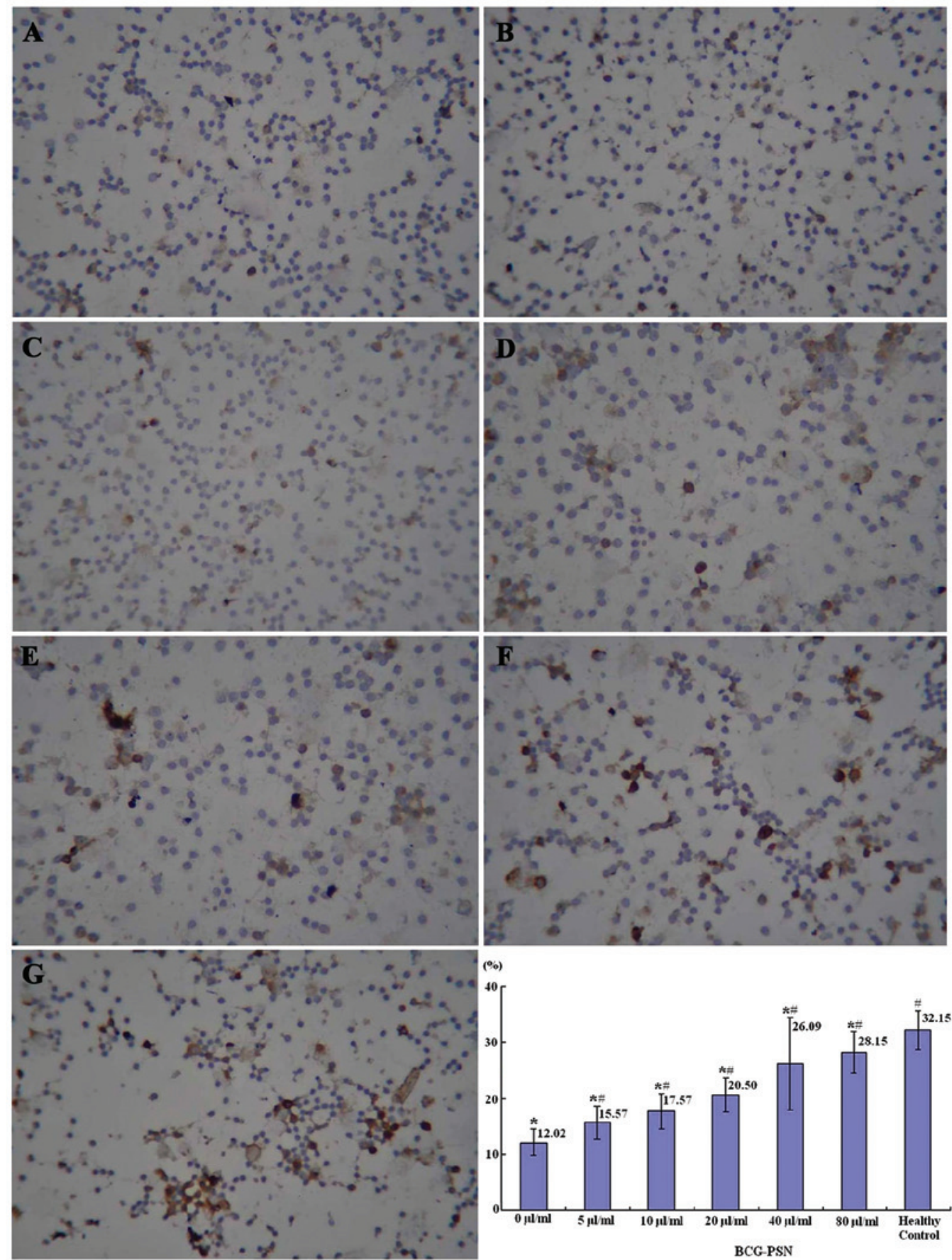

Figure 1. Reduction of IL-2 production by the lymphocytes of patients with chronic idiopathic urticaria (CIU) and enhancement by treatment with the polysaccharide nucleic acid fraction of bacillus Calmette-Guérin (BCG-PSN). After a 96-h incubation, the expression of IL-2 in mononuclear lymphocytes was measured by immunocytochemistry. Error bars indicate standard deviation of the means. "Dunnett's t-test $\mathrm{P}<0.05$, compared to the healthy controls after one-way analysis of variance (ANOVA). "Dunnett's t-test $\mathrm{P}<0.05$, compared to the controls of lymphocytes from CIU patients without BCG-PSN after one-way ANOVA. (A) Lymphocytes of CIU patients without BCG-PSN. Lymphocytes of CIU patients with BCG-PSN in (B) $5 \mu 1 / \mathrm{ml}$, (C) $10 \mu 1 / \mathrm{ml}$, (D) $20 \mu 1 / \mathrm{ml}$, (E) $40 \mu 1 / \mathrm{ml}$ and (F) $80 \mu 1 / \mathrm{ml}$ dilution. (G) Lymphocytes of healthy donors without BCG-PSN.

boosting twice with the AMM vaccine induced a potent antigen-specific interferon (IFN) $\gamma$ and IL-2 production.

Th1 and Th2 are involved in the pathogenesis of CIU, whereas IL-2 and IL-10 are prominent cytokines secreted by Th1 and Th2 cells, respectively. This study was designed to investigate whether the effects of BCG-PSN on the Th1/Th2 balance, determined by its effects on IL-2 and IL-10 production by the lymphocytes of CIU patients, may facilitate the management of CIU.

\section{Materials and methods}

Subjects. This study included 30 CIU patients (15 males and 15 females), aged 18-60 years (average, $38.13 \pm 12$ years), recruited in The Affiliated Hospital of Chengde Medical College (Chengde, China). All the patients met the following selection criteria: age 18-65 years, sharply defined skin wheals persisting for $\geq 6$ weeks, small $(<1 \mathrm{~cm})$ to large $(>8 \mathrm{~cm})$, erythematous or white wheals with an erythematous rim, 


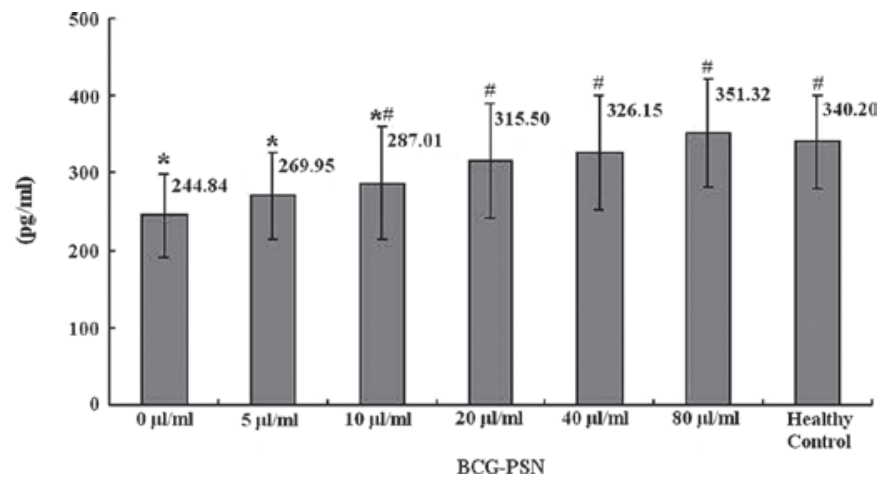

Figure 2. Reduction of IL-2 production by the lymphocytes of patients with chronic idiopathic urticaria (CIU) and enhancement by treatment with the polysaccharide nucleic acid fraction of bacillus Calmette-Guérin (BCG-PSN). After a 96-h incubation, the expression of IL-2 in mononuclear lymphocytes was measured by ELISA. Error bars indicate standard deviation of the means. "Dunnett's t-test $\mathrm{P}<0.05$, compared to the healthy controls after one-way analysis of variance (ANOVA). "Dunnett's t-test $\mathrm{P}<0.05$, compared to the controls of lymphocytes from CIU patients without BCG-PSN after one-way ANOVA.

round, oval, acriform, annular or serpiginous, due to confluence and resolution in one area and progression in another, without definite provocation, pruritic and transient. Patients during pregnancy or lactation, with a history of autoimmune disease or administration of antihistamine drugs, glucocorticosteroids or immunomodulating drugs within the 4 weeks preceding enrollment, were excluded. The 30 controls were healthy donors without any history of allergies.

This study was conducted in accordance with the Declaration of Helsinki and approved by the Ethics Committee of our hospital. All the participants provided written informed consent prior to enrollment.

Immunocytochemistry. Human peripheral blood mononuclear lymphocytes were collected from the venous blood of the subjects by Ficoll-Paque gradient centrifugation. The lymphocytes were adjusted to a final concentration of $2 \times 10^{6} / \mathrm{ml}$ in RPMI-1640 medium with $10 \%$ fetal bovine serum. The lymphocytes in the RPMI-1640 medium (100 $\mu 1$, $\left.2 \times 10^{6} / \mathrm{ml}\right)$ were added to a 96 -well culture plate, in which $10 \mu \mathrm{l}$ of phytohemagglutinin $(2 \mathrm{mg} / \mathrm{ml})$ and differently diluted $(0,5,10,20,40$ and $80 \mu \mathrm{l} / \mathrm{ml})$ of BCG-PSN $(0.35 \mathrm{mg} \mathrm{BCG}$ polysaccharide with $\geq 30 \mu \mathrm{g} / 1$ nucleic acid) had been previously added. The cells were cultured at $37^{\circ} \mathrm{C}$ in a humidified atmosphere containing $5 \% \mathrm{CO}_{2}$ for $96 \mathrm{~h}$. BCG-PSN was obtained from Zhejiang Wanma Pharmaceutical Co., Ltd., Hangzhou, China.

The culture supernatants were collected for ELISA, whereas the cells were smeared on slides and fixed with cold acetone for $5 \mathrm{~min}$. The endogenous peroxidase activity was inactivated with $3 \% \mathrm{H}_{2} \mathrm{O}_{2}$ for $20 \mathrm{~min}$ and blocking was performed for $30 \mathrm{~min}$ in $10 \%$ calf serum. Cells were incubated overnight at $4^{\circ} \mathrm{C}$ with the primary antibody against IL-2 (Santa Cruz Biotechnology Inc., Dallas, TX, USA) or IL-10 (Santa Cruz Biotechnology Inc.), rinsed and incubated with the appropriate horseradish peroxidase (HRP)-conjugated secondary antibody for $30 \mathrm{~min}$ at $37^{\circ} \mathrm{C}$, followed by visualization with diaminobenzidine and counterstaining with hematoxylin prior to mounting. Replacement of the primary antibody with phosphate-buffered saline was used as negative control. The slides were observed under a light microscope. The expression of IL-2 and IL-10 was identified in the cytoplasm and cell membrane as brown colour. The number of positive cells in five separate fields was calculated with a balanced cell number on every slide.

ELISA. The ELISA was performed using the ELISA kit (NeoBioscience, Shenzhen, China) according to the manufacturer's instructions. In brief, the 96-well flat-bottom plate was precoated with anti-human IL-2 or IL-10 antibody. The diluted standards and samples were added to each coated well $\left(100 \mu \mathrm{l} /\right.$ well) and incubated at $36^{\circ} \mathrm{C}$ for $90 \mathrm{~min}$. After 5 washes, biotin-conjugated anti-human IL-2 or IL-10 antibody was added to each well and incubated at $36^{\circ} \mathrm{C}$ for $60 \mathrm{~min}$. After an additional 5 washes, $100 \mu 1$ of HRP-conjugated avidin was added to each well and incubated at $36^{\circ} \mathrm{C}$ in the dark for $30 \mathrm{~min}$. The plate was washed 5 times and $100 \mu \mathrm{l}$ of the tetramethylbenzidine solution was added to the wells, followed by incubation at $36^{\circ} \mathrm{C}$ in the dark for $15 \mathrm{~min}$. The reaction was stopped with $100 \mu \mathrm{l}$ of $2 \mathrm{M}$ sulphuric acid. The optical density was measured at $450 \mathrm{~nm}$ using a microplate reader (Bio-Rad, Hercules, CA, USA). The concentrations of IL-2 and IL-10 (pg/ml) were determined with a standard curve derived from a known amount of the relevant cytokines. The minimum detection level was $8 \mathrm{pg} / \mathrm{ml}$ for IL-2 and $1 \mathrm{pg} / \mathrm{ml}$ for IL-10.

Statistical analysis. The differences between the experimental and control groups were compared with the Pearson's Chi-square test for categorical variables or one-way ANOVA followed by the Dunnett's t-test for continuous variables. The results are presented as percentages for categorical variables, or means $( \pm \mathrm{SD})$ for continuous variables. $\mathrm{P}<0.05$ indicated a statistically significant difference.

\section{Results}

Effects of BCG-PSN on IL-2 production by the lymphocytes of CIU patients. IL-2 is a prominent cytokine secreted by Th1 cells; therefore, it may reflect Th1 function. It was demonstrated by immunocytochemistry that the highest percentage of IL-2-positive lymphocytes was observed in the healthy control group, the lowest in the untreated CIU group $(0 \mu \mathrm{g} / \mathrm{ml}$ BCG-PSN) and there was a sequential increase with the elevation of the BCG-PSN concentration (Fig. 1).

It was also demonstrated by ELISA that the levels of IL-2 in the culture supernatant of the lymphocytes were the highest in the healthy control group, the lowest in the untreated CIU group ( $0 \mu \mathrm{g} / \mathrm{ml}$ BCG-PSN) and there was a sequential increase with the elevation of the BCG-PSN concentration (Fig. 2).

Effects of BCG-PSN on IL-10 production by the lymphocytes of CIU patients. IL-10 is a prominent cytokine secreted by Th 2 cells; therefore, it may reflect Th 2 function. It was demonstrated by immunocytochemistry that the lowest percentage of IL-10-positive lymphocytes was observed in the healthy control group, the highest in the untreated CIU 

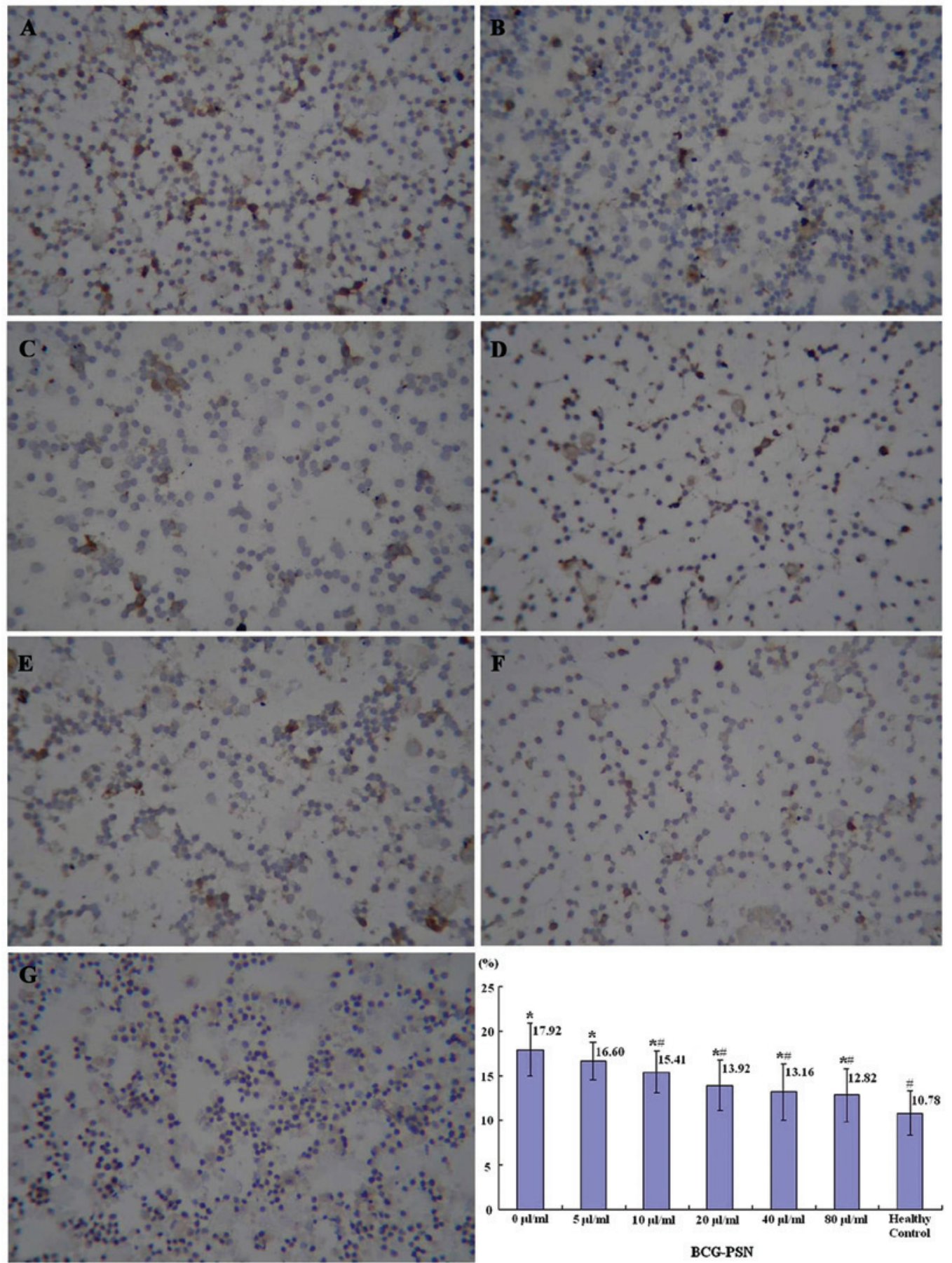

Figure 3. Elevation of IL-10 production by the lymphocytes of patients with chronic idiopathic urticaria (CIU) and reduction by treatment with the polysaccharide nucleic acid fraction of bacillus Calmette-Guérin (BCG-PSN). After a $96 \mathrm{~h}$ incubation, the expression of IL-10 in mononuclear lymphocytes was measured by immunocytochemistry. Error bars indicate standard deviation of the means. "Dunnett's t-test $\mathrm{P}<0.05$, compared to the healthy controls after one-way analysis of variance (ANOVA). "Dunnett's t-test $\mathrm{P}<0.05$, compared to the controls of lymphocytes from CIU patients without BCG-PSN after one-way ANOVA. (A) Lymphocytes of CIU patients without BCG-PSN. Lymphocytes of CIU patients with BCG-PSN in (B) $5 \mu 1 / \mathrm{ml}$, (C) $10 \mu 1 / \mathrm{ml}$, (D) $20 \mu 1 / \mathrm{ml}$, (E) $40 \mu \mathrm{l} / \mathrm{ml}$ and (F) $80 \mu \mathrm{l} / \mathrm{ml}$ dilution. (G) Lymphocytes of healthy donors without BCG-PSN.

group ( $0 \mu \mathrm{g} / \mathrm{ml}$ BCG-PSN) and decreased sequentially with the increase of the BCG-PSN concentration (Fig. 3).

It was also demonstrated by ELISA that the levels of IL-10 in the culture supernatant of the lymphocytes were the lowest in the healthy control group, the highest in the untreated CIU group $(0 \mu \mathrm{g} / \mathrm{ml}$ BCG-PSN $)$ and there was a sequential decrease with the increase of the BCG-PSN concentration (Fig. 4).

\section{Discussion}

$\mathrm{CU}$ causes patients significant physical and mental discomfort. $\mathrm{CU}$ has been defined as a daily or almost daily occurrence of wheals and/or angioedema, occurring over a period of $\geq 6$ weeks (17-19). Furthermore, the direct cost of CU, in terms of healthcare visits, investigation and treatment, is high (20). Several aetiological factors have been associated with the onset 


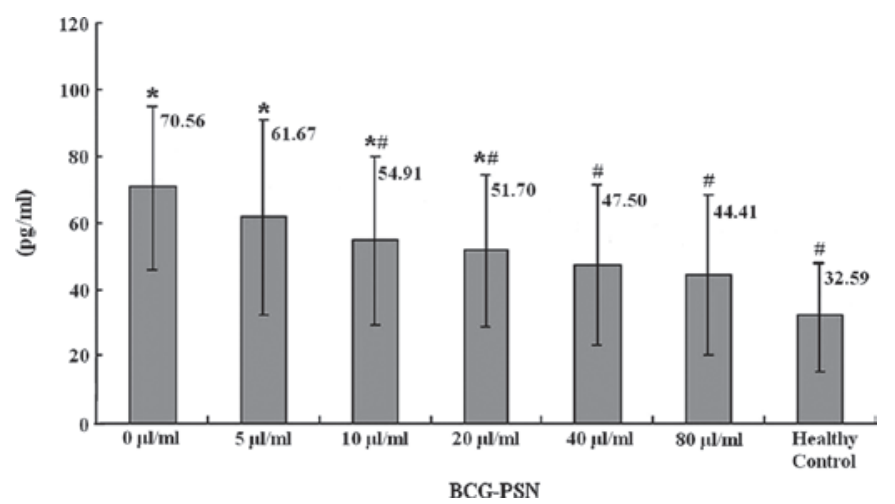

Figure 4. Elevation of IL-10 production by the lymphocytes of patients with chronic idiopathic urticaria (CIU) and reduction by treatment with the polysaccharide nucleic acid fraction of bacillus Calmette-Guérin (BCG-PSN). After a 96-h incubation, the expression of IL-10 in mononuclear lymphocytes was measured by ELISA. Error bars indicate standard deviation of the means *Dunnett's t-test $\mathrm{P}<0.05$, compared to the healthy controls after one-way analysis of variance (ANOVA). "Dunnett's t-test $\mathrm{P}<0.05$, compared to the controls of lymphocytes of CIU without BCG-PSN after one-way ANOVA.

of $\mathrm{CU}$ and it was suggested that the condition has an autoimmune basis $(21,22)$. It was previously observed that IL-10 production was elevated and IL-2 reduced in CIU patients compared to healthy subjects (7). Similar results were demonstrated by the present study, suggesting that the decrease in IL-2 and increase in IL-10 may be an important indicator of the Thl/Th2 imbalance associated with the occurrence and relapse of CIU. Therefore, drugs that may restore the balance between the levels of IL-2 and IL-10 may be an efficient strategy to treat patients with CIU and prevent its recurrence.

BCG-PSN has the ability to regulate the CD4 and CD8 subsets of T cells and the Th1 and Th2 subtypes of helper T cells (23). Following treatment with BCG-PSN, CD4 and CD4/CD8 were significantly increased. In patients with perennial allergic rhinitis, the cellular immunofunction was modulated with an increase of the curative rate (11) and the levels of IL-4 in the serum of patients with condylomata acuminata was reduced following treatment with BCG-PSN (24). The IFN $-\gamma$ and IL-2 production may be induced by BCG (16). It may be concluded that BCG-PSN has the ability to induce T-lymphocyte activation, IFN- $\gamma$ and IL-2 production, IL-4 inhibition and Th1 promotion, thus regulating the Th1/Th2 balance. It is important to elucidate the effect of BCG-PSN on Th1/Th2 balance in the CU patients, due to the potential application of BCG-PSN in the treatment of CU.

$\operatorname{IgE}$ has been frequently associated with CU (25). IL-4 causes a switch to $\mathrm{IgE}$ production through B-cell differentiation; however, IFN- $\gamma$ inhibits that switch, preventing the production of specific IgE (26). IL-2, identified in 1976 as a T-cell growth factor in the supernatants of activated T cells (27), may promote IFN- $\gamma$ production (28). IL-10 is known to downregulate specific effector functions in monocytes, dendritic, natural killer, B and Th1 cells and polymorphonuclear leukocytes (29-31), which may result in the decreased production of pro-inflammatory cytokines and chemokines. IL-10 may inhibit the activity of IFN- $\gamma$, allowing the original IL-4 to proceed in the IgE cascade (26). Therefore, promotion of IL-2 and inhibition of IL-10 production may result in the suppression of IgE production and restore the skin abnormalities in the CIU patients.
In this study, it was demonstrated that the secretion of IL-2 was increased while that of IL-10 was decreased in the peripheral blood lymphocytes of patients with $\mathrm{CIU}$ following treatment with BCG-PSN at different concentrations in vitro for $96 \mathrm{~h}$. In addition, with the increase of drug concentration, the levels of IL-2 and IL-10 were more efficiently restored, suggesting that BCG-PSN may restore the Th1/Th2 imbalance in patients with CIU.

\section{References}

1. Di Campli C, Gasbarrini A, Nucera E, Franceschi F, Ojetti V, Sanz Torre E, Schiavino D, Pola P, Patriarca G and Gasbarrini G: Beneficial effects of Helicobacter pylori eradication on idiopathic chronic urticaria. Dig Dis Sci 43: 1226-1229, 1998.

2. Nagashima Y, Kako K, Kim JD and Fukamizu A: Enhanced histamine production through the induction of histidine decarboxylase expression by phorbol ester in Jurkat cells. Mol Med Rep 6: 944-948, 2012

3. Ouyang H, Shi Y, Liu Z, Feng S, Li L, Su N, Lu Y and Kong S: Increased interleukin-9 and CD4 $4^{+} \mathrm{IL}-9^{+} \mathrm{T}$ cells in patients with systemic lupus erythematosus. Mol Med Rep 7: 1031-1037, 2013.

4. Greaves MW: Chronic idiopathic urticaria. Curr Opin Allergy Clin Immunol 3: 363-368, 2003.

5. Kaplan AP: Chronic urticaria: pathogenesis and treatment. J Allergy Clin Immunol 114: 465-474, 2004.

6. Caproni M, Giomi B, Volpi W, Melani L, Schincaglia E, Macchia D, et al: Chronic idiopathic urticaria: infiltrating cells and related cytokines in autologous serum-induced wheals. Clin Immunol 114: 284-292, 2005.

7. Piconi S, Trabattoni D, Iemoli E, et al: Immune profiles of patients with chronic idiopathic urticaria. Int Arch Allergy Immunol 128: 59-66, 2002.

8. Du Q, Gu X, Cai J, Huang M and Su M: Chrysin attenuates allergic airway inflammation by modulating the transcription factors T-bet and GATA-3 in mice. Mol Med Rep 6: 100-104, 2012.

9. Gu X, Zhou L, Du Q, Jiang D, Yang X, Ji X and Yin K: Hesperetin inhibits the maturation and function of monocyte-derived dendritic cells from patients with asthma. Mol Med Rep 2: 509-513, 2009

10. Sun LX, Lin ZB, Duan XS, Lu J, Ge ZH, Li M, Xing EH, Lan TF, Jiang MM, Yang N and Li WD: Ganoderma lucidum polysaccharides counteract inhibition on CD71 and FasL expression by culture supernatant of B16F10 cells upon lymphocyte activation. Exp Ther Med 5: 1117-1122, 2013.

11. Tang S, Zhao B, Zhang G, Liu L and Zhou T: Immune modulatory and therapeutic effect of BCG polysaccharides nucleic acid on perennial allergic rhinitis. J Clin Otorhinolaryngol 19: 345-346, 2005 (In Chinese).

12. Song NN, Wang BX, Shi DZ, et al: Dimo-thylidioctyl ammonium bromide-BCG polysaccharide nucleic acid adjuvant enhanced the immunogenicity of a Mycobacterium tuberculosis subunit vaccine. Chin J Tuberc Res Dis 32: 513-516, 2009 (In Chinese).

13. Luo Y, Wang B, Hu L, et al: Fusion protein Ag85B-MPT64 (190-198)-Mtb8.4 has higher immunogenicity than Ag85B with capacity to boost BCG-primed immunity against Mycobacterium tuberculosis mice. Vaccine 27: 6179-6185, 2009.

14. Li Q, Yu H, Zhang Y, et al: Immunogenicity and protective efficacy of a fusion protein vaccine consisting of antigen $\mathrm{Ag} 85 \mathrm{~B}$ and HspX against Mycobacterium tuberculosis infection in mice. Scand J Immunol 73: 568-576, 2011.

15. Zhu B, Qie Y, Wang J, et al: Chitosan microspheres enhance the immunogenicity of an Ag85B-based fusion protein containing multiple T-cell epitopes of Mycobacterium tuberculosis. Eur J Pharm Biopharm 66: 318-326, 2007.

16. Luo Y, Jiang W, Da Z, et al: Subunit vaccine candidate AMM down-regulated the regulatory $\mathrm{T}$ cells and enhanced the protective immunity of BCG on a suitable schedule. Scand J Immunol 75: 293-300, 2012.

17. Grattan CEH and Humphreys F; British Association of Dermatologists Therapy Guidelines and Audit Subcommittee: Guidelines for evaluation and management of urticaria in adults and children. Br J Dermatol 157: 1116-1123, 2007. 
18. Zuberbier T, Bindslev-Jensen C, Canonica W, Grattan CEH, Greaves MW, Henz BM, et al: EAACI/GA2LEN/EDF guideline: management of urticaria. Allergy 61: 321-331, 2006.

19. Henz BM and Zuberbier T: Most chronic urticaria is food-dependent, and not idiopathic. Exp Dermatol 7: 139-142, 1998.

20. DeLong KL, Culler Sd, Saini SS, Beck LA and Chen SC: Annual direct and indirect care costs of chronic idiopathic urticaria. Arch Dematol 144: 35-39, 2008.

21. Tong LJ, Balakrishnan G, Kochan JP, Kinet JP and Kaplan AP: Assessment of autoimmunity in patients with chronic urticaria. J Allergy Clin Immunol 99: 461-465, 1997.

22. Gruber BL, Baeza M, Marchese MJ, Agnello V and Kaplan AP: Prevalence and functional role of anti-IgE autoantibodies in urticarial syndromes. J Invest Dermatol 90: 213-217, 1988.

23. Xiong $\mathrm{C}, \mathrm{Li} \mathrm{Q}$, Lin $\mathrm{M}$, et al: The efficacy of topical intralesional BCG-PSN injection in the treatment of erosive oral lichen planus: a randomized controlled trial. J Oral Pathol Med 38: 551-558, 2009.

24. $\mathrm{Hu} \mathrm{J}$ and Chen H: The effect of BCG-PSN on T-cell subsets and cytokines in vernal conjunctivitis. J Huazhong Univ Sci Technolog Med Sci 22: 77-79, 2002.

25. Du Toit G, Prescott R, Lawrence P, Johar A, Brown G, Weinberg EG, et al: Autoantibodies to the high-affinity $\operatorname{IgE}$ receptor in children with chronic urticaria. Ann Allergy Asthma Immunol 96: 341-344, 2006.
26. Kidd P: Th1/Th2 balance: the hypothesis, its limitations, and implications for health and disease. Altern Med Rev 8: 223-246, 2003.

27. Morgan DA, Ruscetti FW and Gallo R: Selective in vitro growth of T lymphocytes from normal human bone marrows. Science 193: 1007-1008, 1976.

28. Bird JJ, Brown DR, Mullen AC, Moskowitz NH, Mahowald MA, Sider JR, Gajewski TF, Wang CR and Reiner SL: Helper T cell differentiation is controlled by the cell cycle. Immunity 9: 229-237, 1998.

29. Cassatella MA, Gasperini S, Bovolenta C, Calzetti F, Vollebregt M, Scapini P, et al: Interleukin-10 (IL-10) selectively enhances CIS3/SOCS3 mRNA expression in human neutrophils: evidence for an IL-10-induced pathway that is independent of STAT protein activation. Blood 94: 2880-2889, 1999.

30. Moore KW, O'Garra A, de Waal Malefyt R, Vieira P and Mosmann TR: Interleukin-10. Annu Rev Immunol 11: 165-190, 1993.

31. Buelens C, Willems F, Delvaux A, Piérard G, Delville JP, Velu $\mathrm{T}$ and Goldman M: Interleukin-10 differentially regulates B7-1 (CD80) and B7-2 (CD86) expression on human peripheral blood dendritic cells. Eur J Immunol 25: 2668-2672, 1995. 